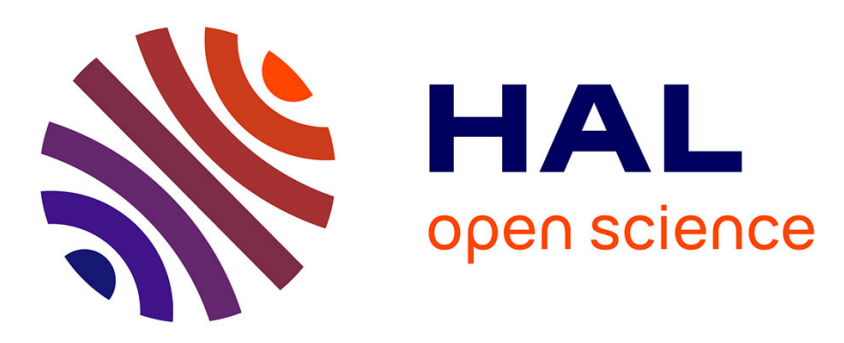

\title{
Local Structure-Driven Localized Surface Plasmon Absorption and Enhanced Photoluminescence in ZnO-Au Thin Films
}

William Chamorro, Jaafar Ghanbaja, Yann Battie, Aotmane En Naciri, Flavio Soldera, Frank Mücklich, David Horwat

\section{To cite this version:}

William Chamorro, Jaafar Ghanbaja, Yann Battie, Aotmane En Naciri, Flavio Soldera, et al.. Local Structure-Driven Localized Surface Plasmon Absorption and Enhanced Photoluminescence in ZnO-Au Thin Films. Journal of Physical Chemistry C, 2016, 120 (51), pp.29405-29413. 10.1021/acs.jpcc.6b09974 . hal-02899484

\section{HAL Id: hal-02899484 \\ https://hal.univ-lorraine.fr/hal-02899484}

Submitted on 5 Jul 2021

HAL is a multi-disciplinary open access archive for the deposit and dissemination of scientific research documents, whether they are published or not. The documents may come from teaching and research institutions in France or abroad, or from public or private research centers.
L'archive ouverte pluridisciplinaire HAL, est destinée au dépôt et à la diffusion de documents scientifiques de niveau recherche, publiés ou non, émanant des établissements d'enseignement et de recherche français ou étrangers, des laboratoires publics ou privés. 


\title{
Local Structure-Driven Localized Surface Plasmon Absorption and Enhanced Photoluminescence in ZnO-Au Thin Films
}

\author{
William Chamorro, ${ }^{\dagger}$, Jaafar Ghanbaja, ${ }^{\dagger}$ Yann Battie, ${ }^{\dagger}$ Aotmane En Naciri, ${ }^{\ddagger}$ Flavio Soldera, ${ }^{\S}$ \\ Frank Mücklich, ${ }^{\S}$ and David Horwat* ${ }^{\dagger} \dagger$ \\ ${ }^{\dagger}$ Université de Lorraine, Institut Jean Lamour, UMR7198, Nancy, F-54011, France \\ ${ }^{\ddagger}$ LCP-A2MC, Institut Jean Barriol, Université de Lorraine, 1 Bd Arago, 57070 Metz, France \\ ${ }^{\S}$ Department Materials Science and Engineering, Saarland University, D-66123 Saarbrücken, Germany
}

\author{
Supporting Information
}

\begin{abstract}
Nanocomposite films consisting of gold nanoparticles embedded in zinc oxide ( $\mathrm{ZnO}-\mathrm{Au})$ have been synthesized with different gold loadings by reactive magnetron sputtering at near-room temperature followed by ex situ annealing in air up to $300{ }^{\circ} \mathrm{C}$. Using X-ray diffraction and high resolution transmission microscopy it is shown that during deposition gold substitutes zinc in $\mathrm{ZnO}$ as isolated atoms and in nanoparticles still exhibiting the structure of $\mathrm{ZnO}$. Both situations degrade the crystalline quality of the $\mathrm{ZnO}$ matrix, but thermal annealing cures it from isolated gold atoms and triggers the formation of gold nanoparticles of size higher than $3 \mathrm{~nm}$, sufficient to observe a strong activation of localized surface plasmon resonance (LSPR). The amplitude of LSPR absorption observed after annealing increases with the gold

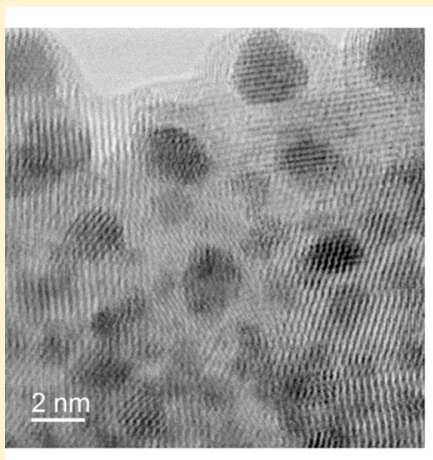
Energy (eV)

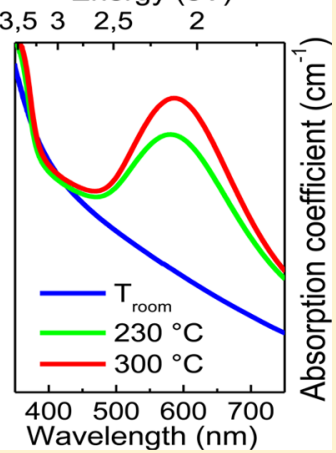
loading and annealing temperature. Moreover, UV and visible photoluminescence from the $\mathrm{ZnO}$ matrix is strongly enhanced upon activation of LSPR showing strong coupling with the gold nanoparticles. Finally, modeling of spectroscopic ellipsometry measurements unambiguously reveals how curing the defects increases the optical bandgap of the $\mathrm{ZnO}$ matrix and modifies the optical dielectric functions of the nanocomposite and $\mathrm{ZnO}$ matrix.
\end{abstract}

\section{INTRODUCTION}

Since the early 1990s, the optical properties of gold nanoparticles (NPs) have attracted renewed interest due to the rapid development of plasmonics as an independent extremely active field of research and applications. ${ }^{1}$ Effectively, gold NPs exhibit localized surface plasmon resonance (LSPR). LSPR consists in resonant coupling between photons and the collective oscillation of metal conduction electrons of the same frequency (the plasmon frequency, $\omega_{\mathrm{r}}$ ) at the surface of metal nanoparticles. The nanoparticles act as nanoantennas. They induce several effects such as optical absorption, the concentration of electric field, electric near field enhancement in the direct surrounding of the nanoparticles, and heating of the nanoparticles upon excitation by photons of $\omega_{\mathrm{r}}$. To date, most of the efforts have been focused on producing colloidal NPs by chemical routes. However, colloidal NPs tend to grow by Ostwald ripening, even at moderate temperature, and to agglomerate. Since the amplitude and energy of the LSPR absorption is highly sensitive to the size of NPs, excessive growth and agglomeration often leads to loss or weakening of the LSPR absorption and related properties. These problems can be solved by stabilizing the size and shape of the NPs, thanks to chemical interaction with another material. This is referred as "nanocomposite" architectures. Different possible configurations of metal-semiconductor plasmonic nanocomposites can be found: core-shell nanostructures, ${ }^{2}$ metal supported on semiconducting surface ${ }^{3}$ (outside structure) or metal embedded in a semiconducting matrix ${ }^{4}$ (inside structure). There is a growing interest in using semiconducting oxides in contact to plasmonic NPs in order to benefit from LSPR-enhanced optoelectronic properties. Zinc oxide is a particularly interesting multifunctional $n$-type wide bandgap semiconductor showing electronic conductivity close to $10^{-4} \mathrm{~S}$. $\mathrm{cm}^{-1}$ when heterovalent ions substitute the $\mathrm{Zn}$ site. ${ }^{5}$ Moreover, due to its large exciton binding energy, $\mathrm{ZnO}$ can emit ultraviolet light following exciton recombination (near band edge emission, NBE) at room temperature. It also exhibits emission in the visible range related to the presence of point defects, ${ }^{6}$ as well as tunable optical absorption in the ultraviolet and near-infrared regions. ${ }^{7}$ Therefore, coupling gold NPs to $\mathrm{ZnO}$ within nanocomposite architectures represents an interesting approach to design multifunctional surfaces

Received: October 2, 2016

Revised: November 24, 2016

Published: December 5, 2016 
combining the properties of the constituents as well as new properties arising from $\mathrm{Au}-\mathrm{ZnO}$ interactions. $\mathrm{ZnO}-\mathrm{Au}$ nanocomposites have been studied for enhanced UV photodetection, ${ }^{8}$ gas sensing, ${ }^{8-10}$ and plasmon catalysis, ${ }^{11,12}$ and are foreseen as efficient absorbers for photovoltaic solar cells. ${ }^{13}$ Recent studies indicate the possibility to inject "hot electrons" in semiconductors or molecules in contact with plasmonic nanoparticles ${ }^{14,15}$ based on photoelectron emission by nonradiative electromagnetic decay after LPSR excitation. Interestingly, $\mathrm{ZnO}$ has suitably positioned valence and conduction bands to optimize "hot electrons" injection from plasmonic nanoparticles. New concepts of photodiodes ${ }^{14}$ and solar cells ${ }^{13}$ based on this phenomenon have been proposed. To date the efficiencies of such devices are very low but these technologies are in their infancy and strategies to improve it have already emerged. For instance, it has been calculated that engineering of the Schottky barrier established at the metal-semiconductor interface and modification of the charge carrier density at the plasmonic particles could enhance the efficiency of this new type of solar cells well in excess of $22 \%$, that is, rendering it competitive with conventional solar cells but with much higher light trapping efficiency. ${ }^{16}$ Knight et al. showed that electron transfer is enhanced at embedded junctions. ${ }^{17}$ Therefore, plasmonic $\mathrm{ZnO}-\mathrm{Au}$ nanocomposites, enabling charge transfer at the AulZnO interface are highly sought, but there is little knowledge on the influence of the synthesis and treatment conditions on the local properties of such nanocomposites.

Several methods have been employed for the synthesis of $\mathrm{ZnO}-\mathrm{Au}$ nanocomposites films such as electrodeposition, magnetron sputtering, and pulsed laser deposition. ${ }^{18-20}$ In this work, we use reactive magnetron sputtering for three main reasons. First, it enables a precise control of the film chemical composition; which can be of primary importance to understand the origin of property modifications. Second, the microstructure and optical properties of $\mathrm{ZnO}$ thin films can be controlled through the experimental parameters such as the $\mathrm{Ar}: \mathrm{O}_{2}$ reactive atmosphere composition or using substrates to stimulate epitaxial growth. ${ }^{6}$ Finally, the scalability of the method is demonstrated and transfer of results gained at the laboratory scale to the industry is straightforward in most cases. In this report, we describe the synthesis and characterization of $\mathrm{ZnO}-\mathrm{Au}$ nanocomposite films grown by reactive DC magnetron cosputtering. More particularly, it is shown how the gold content and thermal treatment influence the film microstructure and, thereby, the optical properties of the films, including LSPR absorption by gold NPs and enhanced photoluminescence in UV and visible ranges.

\section{EXPERIMENTAL SECTION}

2.1. Synthesis. $\mathrm{ZnO}$ and $\mathrm{ZnO}-\mathrm{Au}$ nanocomposite thin films have been grown on silica and [0001]-oriented sapphire by reactive DC magnetron cosputtering of $\mathrm{Au}$ and $\mathrm{Zn}$ targets (50 $\mathrm{mm}$ diameter, $3 \mathrm{~mm}$ thickness, and purities over $99.9 \%$ ). The target-substrate distance was set to 50 and $55 \mathrm{~mm}$ for the $\mathrm{Zn}$ and Au targets, respectively, and the targets were placed off-axis relatively to the rotating substrate-holder axis in order to ensure a good homogeneity in thickness and composition. ${ }^{21}$ The samples were positioned on the circumference of the substrateholder facing the magnetron axis. The argon flow rate was fixed to 50 standard cubic centimeters per minute $(\mathrm{sccm})$ and the oxygen flow rate (OFR) was kept constant at $8 \mathrm{sccm} \mathrm{O}_{2}$, corresponding to an injected composition of the gas mixture of 14 vol $\% \mathrm{O}_{2}$ and a total pressure close to $0.5 \mathrm{~Pa}$. We showed in a recent report that an OFR of $8 \mathrm{sccm}$ using the same experimental procedure leads to $\mathrm{ZnO}$ films grown in the zincrich region. ${ }^{6}$ The surface of the samples was activated in situ using radiofrequency polarization $(60 \mathrm{~W}, 2 \mathrm{~min})$ in the reactive ambiance just before deposition. The discharge current for the $\mathrm{Zn}$ target was fixed to $0.07 \mathrm{~A}$. This low discharge current was used to prevent substantial self-heating of the film during bombardment by fast particles impinging the substrates after their production at, or reflection by, the target surface. For the $\mathrm{ZnO}-\mathrm{Au}$ nanocomposite films, the atomic gold content is defined as $(\mathrm{Au}) /(\mathrm{Au}+\mathrm{Zn}) \times 100$. To vary the Au composition between the different experiments, the $\mathrm{Au}$ target density current was varied from 0.015 to $0.006 \mathrm{~A}$ (the lowest reachable current for the Au target) using an MDX 500 advanced energy supply. The substrate temperature was evaluated using thermal level stripes (Thermax) positioned on the same circumference as the substrates on the substrate-holder and was below $40{ }^{\circ} \mathrm{C}$ (the lower detection limit of the thermal level stripes) for all conditions. The deposition time was set such that the nanocomposite grown films have a thickness of $150 \pm 40$ $\mathrm{nm}$, whatever the chemical composition, as measured using tactile profilometry. The influence of annealing in air at 230 and $300{ }^{\circ} \mathrm{C} \pm 5{ }^{\circ} \mathrm{C}$ for $1 \mathrm{~h}$ on the microstructure and properties of $\mathrm{ZnO}-\mathrm{Au}$ films was studied.

2.2. Characterizations. Optical transmittance measurements were performed with a Cary 5000 UV-vis-NIR spectrophotometer. The film structure and microstructure were characterized by $\theta-2 \theta \mathrm{X}$-ray diffraction (XRD) measurements with a AXS Bruker D8 Advance diffractometer using a $\mathrm{Cu}$ anode $(\mathrm{Cu} \mathrm{K} \alpha=1.54 \AA)$. Further details on the microstructure were obtained with scanning transmission electron microscopy (STEM) investigations performed using a Philips CM200 Microscope and a JEOL ARM 200-Cold FEG microscope. The working voltage of the microscopes is close of $200 \mathrm{kV}$. For this purpose, TEM foils were prepared in a focused ion beam (FIB)/scanning electron microscope (SEM) dual beam system (FEI Helios 600) by using the "in situ" lift-out technique. Thinning of the foil was done using acceleration voltage of $30 \mathrm{kV}$ at the first steps and finishing with $5 \mathrm{kV}$ and a current of $16 \mathrm{pA}$ in order to reduce possible artifacts and amorphization. The chemical composition was determined by energy dispersive X-ray spectroscopy (EDS) in the SEM.

A mechanical strain analysis was conducted by coupling the curvature method ${ }^{22}$ and interpretation of XRD data.

The optical properties of $\mathrm{ZnO}$ and $\mathrm{ZnO}-\mathrm{Au}$ films were deduced from ellipsometric measurements by using a phase modulation ellipsometer (UVISEL, Horiba Jobin Yvon). The ellipsometric parameters $I_{\mathrm{s}}$ and $I_{\mathfrak{c}}$, which depend on the ellipsometric angles $\Psi$ and $\Delta$, are given by

$$
\begin{aligned}
& I_{\mathrm{s}}=\sin 2 \psi \sin \Delta \\
& I_{\mathrm{c}}=\sin 2 \psi \cos \Delta
\end{aligned}
$$

Both parameters were measured and fitted in the $260-825 \mathrm{~nm}$ spectral range at several angles of incidences from $50^{\circ}$ to $70^{\circ}$ (see Supporting Information). Ellipsometric measurements recorded on pure $\mathrm{ZnO}$ films were analyzed by modeling samples as a two-layer structure on a silicon substrate. The bottom layer and top layer corresponds to the $\mathrm{ZnO}$ film and the film roughness, respectively. The Tanguy dispersion was used to determine the dielectric function of $\mathrm{ZnO}\left(\varepsilon_{\mathrm{ZnO}}\right):^{23}$ 


$$
\begin{aligned}
\varepsilon & =\varepsilon_{\infty}+\frac{a}{b-\omega^{2}}+\frac{A \sqrt{R}}{(\omega+i \Gamma)^{2}}\left(\frac{\ln \left(\omega_{\mathrm{g}}^{2}\right)}{\omega_{\mathrm{g}}^{2}-(\omega+i \Gamma)^{2}}+2 \sqrt{\frac{\omega_{\mathrm{g}}}{R}}\right. \\
& -\sqrt{\frac{\omega_{\mathrm{g}}+\omega+i \Gamma}{R}}+2 \sum_{k=1}^{\infty}\left(\frac{2}{\sqrt{\frac{R}{\omega_{\mathrm{g}}}}-k}-\frac{1}{\sqrt{\frac{R}{\omega_{\mathrm{g}}+\omega+i \Gamma}}-k}\right. \\
& \left.\left.-\frac{1}{\sqrt{\frac{R}{\omega_{\mathrm{g}}-\omega-i \Gamma}}-k}\right)\right)
\end{aligned}
$$

where $R$ is the exciton binding energy, $A$ the amplitude factor, $\omega_{\mathrm{g}}$ the energy bandgap, and $\Gamma$ the damping factor. A first Sellmeier term has also added to account for higher energy transitions.

The dielectric function of the film roughness $\left(\varepsilon_{\text {rough }}\right)$ is described by the Bruggeman effective model by considering a mixture of $50 \%$ of voids and $50 \%$ of $\mathrm{ZnO}$ :

$$
0=\frac{1-\varepsilon_{\text {rough }}}{1+2 \varepsilon_{\text {rough }}}+\frac{\varepsilon_{\mathrm{ZnO}}-\varepsilon_{\text {rough }}}{\varepsilon_{\mathrm{ZnO}}+2 \varepsilon_{\text {rough }}}
$$

Ellipsometric measurements performed at four angles of incidences were simultaneously fitted by adjusting the parameters of the $\mathrm{ZnO}$ dispersion law $\left(\varepsilon_{\infty}, A, E_{\mathrm{g}}, R, \Gamma, a, b\right)$, the thicknesses of $\mathrm{ZnO}$ film, and the thickness of the roughness layer. The root-mean-square error between the measurements and the fit was equal to 0.1. Experimental and best fit to the data are presented in the Supporting Information. $\mathrm{ZnO}-\mathrm{Au}$ nanocomposites have also been characterized by spectroscopic ellipsometry. The ellipsometric data were analyzed by considering the same two-layer structure as for pure $\mathrm{ZnO}$ but it was necessary to consider that the optical response is the contribution of all components. We used the Maxwell-Garnett (MG) effective medium model to obtain the effective dielectric function of $\mathrm{ZnO}-\mathrm{Au}$ films $\left(\varepsilon_{\mathrm{ZnO}-\mathrm{Au}}\right)$. The $\mathrm{MG}$ considers the presence of inclusions in a host matrix. This model is most used when the volume fraction $(f)$ of the inclusions is low enough to consider the particles are well separated. The $\mathrm{ZnO}-\mathrm{Au}$ nanocomposite films prepared for this study exhibit such behavior. The Maxwell-Garnett effective medium model relates the dielectric constants by

$$
\frac{\varepsilon_{\mathrm{ZnO}-\mathrm{Au}}-\varepsilon_{\mathrm{ZnO}}}{\varepsilon_{\mathrm{ZnO}-\mathrm{Au}}+2 \varepsilon_{\mathrm{ZnO}}}=f \frac{\varepsilon_{\mathrm{Au}}-\varepsilon_{\mathrm{ZnO}}}{\varepsilon_{\mathrm{Au}}+2 \varepsilon_{\mathrm{ZnO}}}
$$

where $\varepsilon_{\mathrm{ZnO}}, \varepsilon_{\mathrm{Aw}}$ and $\varepsilon_{\mathrm{ZnO}-\mathrm{Au}}$ are the dielectric constants of the $\mathrm{ZnO}$ matrix, gold NPs, and the effective dielectric function of the film, respectively. The dielectric function of $\mathrm{ZnO}$ was calculated from the Tanguy dispersion law (eq 1). The gold dielectric function is the sum of two contributions due to the interband $\left(\varepsilon_{\text {inter }}\right)$ and intraband $\left(\varepsilon_{\text {intra }}\right)$ transitions:

$$
\varepsilon_{\text {Au }}=\varepsilon_{\text {inter }}+\varepsilon_{\text {intra }}
$$

The contribution of intraband transitions is described by the Drude relations.

$$
\varepsilon_{\text {intra }}=\varepsilon_{\infty}+\frac{\omega_{\mathrm{p}}^{2}}{\omega(\omega+i \Gamma)}
$$

where $\omega_{\mathrm{p}}, \Gamma$, and $\varepsilon_{\infty}$ are the plasma frequency, damping factor and the dielectric constant at high energy, respectively. The contribution of interband contribution was modeled by using a Tauc-Lorentz dispersion law with three oscillators.

$$
\varepsilon_{\text {inter }, \mathrm{i}}=\left\{\begin{array}{l}
\sum_{k=1}^{3} \frac{1}{\omega} \frac{A_{k} \omega_{k} C_{k}\left(\omega-\omega_{\mathrm{th}}\right)^{2}}{\left(\omega-\omega_{k}^{2}\right)^{2}+C_{k}^{2} \omega^{2}} \text { for } \omega>\omega_{\mathrm{th}} \\
0 \text { for } \omega \leq \omega_{\mathrm{th}}
\end{array}\right.
$$

and

$$
\varepsilon_{\text {inter }, \mathrm{r}}=\frac{2}{\pi} \int_{\omega_{\text {th }}}^{\infty} \frac{\Omega \varepsilon_{1 \mathrm{i}}}{\Omega^{2}-\omega^{2}} \mathrm{~d} \Omega
$$

where $\varepsilon_{\text {inter,r }}$ and $\varepsilon_{\text {inter,i }}$ are the real part and imaginary part of $\varepsilon_{\text {inter }} ; \omega_{\text {th }}$ is the threshold energy of the interband transitions, while $\omega_{k}, c_{k}$, and $A_{k}$ are the energy, the broadening, and the strength of the transition $k$, respectively. Each parameter in $\varepsilon_{\mathrm{Au}}$ are set to the ones estimated by fitting the tabulated dielectric function of bulk Au. ${ }^{24}$ For nanoparticle radius smaller than 5 $\mathrm{nm}$, the confinement effect has a strong impact on the Drude part of Au dielectric function. ${ }^{25}$

The ellipsometric data were fitted by using a two steps procedure. In the first step, the dielectric function of the $\mathrm{ZnO}$ matrix was set to the measured one on pure $\mathrm{ZnO}$ film. The Drude part of the dielectric function of Au NPs $\left(\varepsilon_{\text {intra }}\right)$, their volume fraction and the thicknesses of the roughness and $\mathrm{ZnO}$ $\mathrm{Au}$ layers were simultaneously fitted. Then, these parameters were set to obtained values. In the second step, we only fit the dielectric function of the $\mathrm{ZnO}$ matrix. Both steps were repeated until a convergence was achieved. The root-mean-square error between the measurements and the fit was equal to 0.1 . Experimental data and best fit to the data are presented in the Supporting Information.

Photoluminescence (PL) measurements have been performed on selected films using laser excitation at $325 \mathrm{~nm}$.

\section{RESULTS AND DISCUSSION}

3.1. Film Structure and Microstructure. In order to evaluate the influence of the gold loading on the microstructure of the films, the gold content, defined as $(\mathrm{Au}) /(\mathrm{Au}+\mathrm{Zn}) \times$ 100, has been varied between 10 and 29\%. As shown in Figure 1 , in as-deposited samples, the (0002) $\mathrm{ZnO}$ diffraction peak is

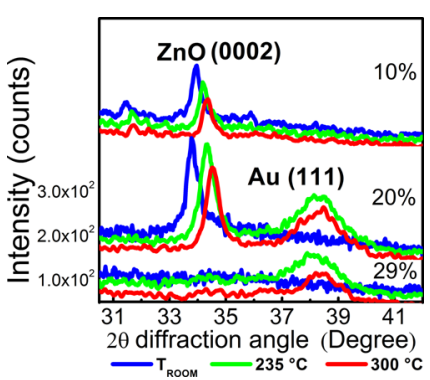

Figure 1. X-ray diffractograms of $\mathrm{ZnO}$-Au nanocomposite thin films synthesized with different Au contents (10,20, and 29\%) as-deposited $\left(T_{\text {Room }}\right)$ and after annealing in air at 230 and $300{ }^{\circ} \mathrm{C} \pm 5{ }^{\circ} \mathrm{C}$.

detected up to $20 \% \mathrm{Au}$. However, the diffracted intensity is weak compared to pure $\mathrm{ZnO}$, about 2 orders of magnitude lower (see Supporting Information), and no diffraction signal of crystalline $\mathrm{ZnO}$ is detected at the highest gold loading of $29 \%$.

Hence, the incorporation of gold has a marked influence on the film microstructure. Several processes can be at the origin of this observation. First, $\mathrm{Au}$ atoms may be present in the $\mathrm{ZnO}$ matrix, as suggested by recent investigations on RF sputterdeposited $\mathrm{ZnO}-\mathrm{Au}$ films, ${ }^{26}$ which can induce local strain and, 
thereby, degrade the crystallinity of $\mathrm{ZnO}$. Second, impurities can interfere with crystal growth. ${ }^{27}$ Such impurities could be present in the form of isolated or clustered gold atoms. The broad signal in the region $37-39.5^{\circ}$ in the diffractogramms of samples containing 20 and $29 \%$ Au annealed in air corresponds to the diffraction by (111) planes of crystalline Au. The large width indicates the nanocrystalline character of gold. This signal is neither observed in as-deposited samples nor after annealing for the lowest gold content, suggesting that before annealing gold atoms are distributed in the $\mathrm{ZnO}$ matrix. This hypothesis will be tested in the following. Further analysis of the microstructure was conducted using high resolution transmission microscopy in scanning transmission electron microscope (STEM) mode. Bright field (BF) and high angle annular dark field (HAADF: $Z$ contrast) micrographs of asdeposited film with $10 \% \mathrm{Au}$ are shown on Figure 2a,b. Heavy
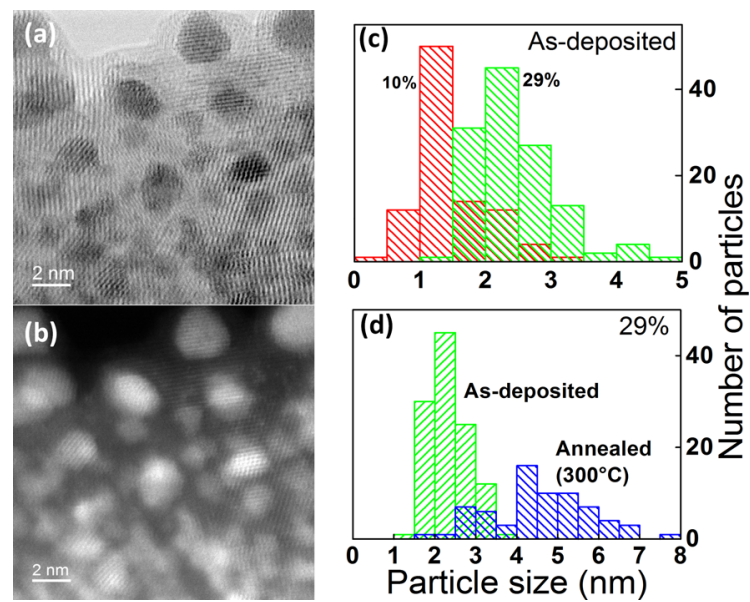

Figure 2. Bright field (a) and high angle annular dark field (b) STEM micrographs of a $\mathrm{ZnO}-\mathrm{Au}$ nanocomposite film with $10 \%$ gold. Distributions of nanoparticle size (c) for two gold contents in the asdeposited state (up) and for $29 \%$ gold in the as-deposited state and after annealing at $300{ }^{\circ} \mathrm{C}$ in air (bottom).

atoms appear in dark and clear in the BF and HAADF images, respectively. Precipitates involving heavy atoms, obviously gold, are clearly visible in a matrix constituted by lighter atoms. Nevertheless, the interplanar distances of fcc gold were not detected in the images and in the selected area electron diffraction dominated by the signal of crystalline $\mathrm{ZnO}$ (see Supporting Information). Moreover, a close look at the images indicates the continuity between the crystal lattice of precipitates and that of the $\mathrm{ZnO}$ matrix. A distance close to $0.27 \mathrm{~nm}$ is measured between nearest neighbors in dark particles showing coherence with the basal plane of the hexagonal $\mathrm{ZnO}$ matrix. This does not correspond to a characteristic distance of the fcc gold crystal structure. This is consistent with the XRD measurements supporting our hypothesis that all gold atoms are distributed in the $\mathrm{ZnO}$ matrix before annealing. In contrast, annealing the samples in air induces the precipitation of crystalline fcc gold NPs as detected in the X-ray diffractograms for the two highest loadings and in the selected area electron diffraction pattern (see Supporting Information). The particle size distribution was extracted for various gold contents before and after annealing from $\mathrm{BF}$ and HAADF images. The main results are summarized in Figure 2c. It is found that the gold loading increases the average size of NPs, similarly with previous observations on the yttria stabilized zirconia-gold system; ${ }^{28}$ so does the thermal annealing in air. Our experience with Au-YSZ led us to limit the gold loading to $29 \%$ in order to prevent substantial migration of gold to the film-air interface during annealing due to eased diffusion within a percolating network of gold NP. ${ }^{28}$ The annealing treatment was therefore sufficient to trigger the reorganization of gold atoms in crystalline gold NPs. The average size of nanoparticles ranges from $\sim 1.5 \mathrm{~nm}$ for the lowest gold loading before annealing to $\sim 4.5 \mathrm{~nm}$ for the sample with highest gold loading after annealing in air.

The (0002) $\mathrm{ZnO}$ diffraction peak of Figure 1 shifts toward lower angles with increasing the Au content and the annealing temperature, indicating a variation of the $c$-axis parameter. We can follow the evolution of the $\mathrm{ZnO} c$-axis parameter with the $\mathrm{Au}$ content and the annealing temperature in the contour map of Figure 3a.

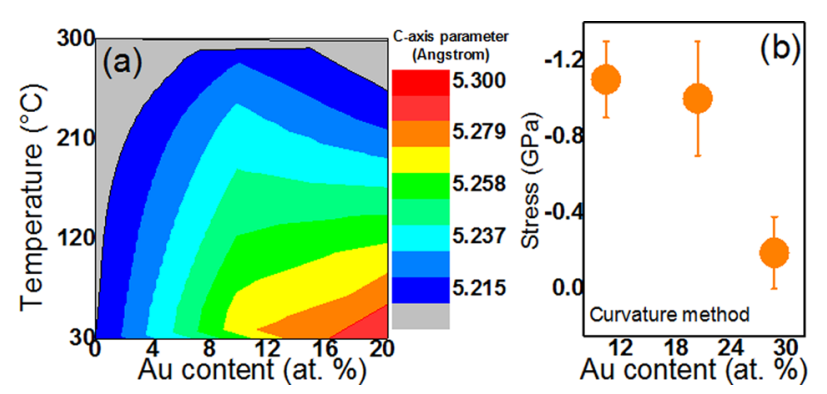

Figure 3. $2 \mathrm{D}$ contour plot of the $c$-axis parameter determined using the (0002) $\mathrm{ZnO}$ diffraction peak of $\mathrm{ZnO}$ and $\mathrm{ZnO}-\mathrm{Au}$ nanocomposite films as a function of the temperature and the gold content (a). Stress measurements using the curvature method for $\mathrm{ZnO}-\mathrm{Au}$ nanocomposite films as a function of the Au content (b).

It is found that increasing the gold content and the annealing temperature have opposite effects as it increases and decreases the $c$-axis parameter, respectively. Indeed, the annealing treatment enables reaching a $c$-axis parameter close to that of a pure bulk $\mathrm{ZnO}$ (5.2042 $\mathrm{\AA}$ ) for all gold contents but requires a different holding temperature depending on the gold content. There are several possible explanations to these observations. Within the $\mathrm{ZnO}$ biaxial in-plane strain model, ${ }^{29}$ the elongation (contraction) of the $c$-axis parameter can be interpreted as inplane compressive (tensile) stress state. In order to verify the validity of this model in the present situation, stress measurements have also been performed using the curvature method $^{22}$ (Figure 3b). All as-deposited films exhibit a compressive stress state but, in contradiction with the biaxial in-plane strain interpretation of the data of Figure 3a, the amplitude of the compressive stress decreases as the gold content is increased. Compressive stresses almost disappear at $29 \% \mathrm{Au}$. To solve this apparent contradiction it is necessary to consider that the curvature method gives global information about the film in-plane stress state, while the position of the (0002) diffraction peak gives information on the strain in the matrix only. It is well-known that Au exhibits a ductile behavior. Hence, due to the contribution of "soft" particles containing $\mathrm{Au}$, the Young modulus of $\mathrm{ZnO}-\mathrm{Au}$ nanocomposite films is expected to be lower than that of the $\mathrm{ZnO}$ matrix alone (111 $\mathrm{GPa}),{ }^{30}$ as observed with the YSZ-Au system. ${ }^{31}$ As mentioned earlier, a possible origin for the degradation of the crystallinity of the $\mathrm{ZnO}$ matrix is the presence of gold atoms in the crystal lattice of $\mathrm{ZnO}$, as reported recently for RF sputter-deposited $\mathrm{ZnO}-\mathrm{Au}$ films and suggested by the high resolution STEM 
results on our as-deposited films. More precisely, the gold atoms were found to substitute zinc atoms. ${ }^{26}$ These "trapped" atoms expand the $\mathrm{ZnO}$ lattice due to their larger radius than $\mathrm{Zn}$ atoms. Based on that hypothesis, the deformation of the $c$-axis parameter brings information about the probability to find "trapped" Au atoms in the $\mathrm{ZnO}$ matrix. The thermal evolution can thereby be explained by the release of $\mathrm{Au}$ atoms from the $\mathrm{ZnO}$ structure and their diffusion to form Au NPs. Hence, the evolutions of the $c$-axis and stress state, as measured by the curvature method, are not contradictory, but give different information on the influence of distributed gold atoms on the microstructure and mechanical properties of the films, respectively.

Figure 4 is a magnification of the STEM bright field image of Figure 2 collected before annealing. The contrast was modified

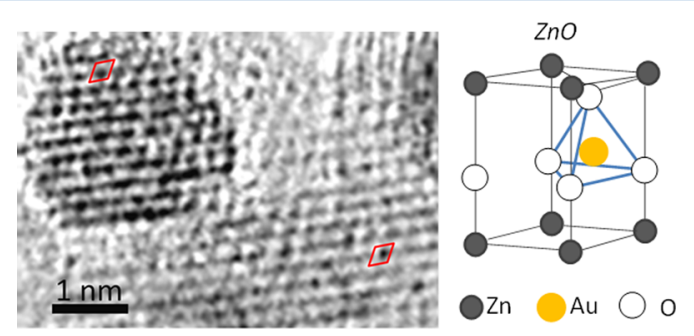

Figure 4. Magnified view of the STEM bright field image of Figure 2 and corresponding interpretation of the positioning of gold atoms in the $\mathrm{ZnO}$ crystal lattice and at NPs before annealing.

in order to better highlight the presence of heavy atoms, obviously gold ones. These atoms are located in nanoparticles and as isolated atoms in the $\mathrm{ZnO}$ matrix. They introduce visible distortion. Moreover, the structure at nanoparticles appears to be derived from that of $\mathrm{ZnO}$ in which a large density of gold atoms has been introduced. At some locations both in the matrix and at gold NPs, it is possible to retrieve the basal plane of the $\mathrm{ZnO}$ hexagonal lattice containing a projected position of gold atom at coordinates corresponding to $1 / 3$ and $2 / 3$ the a lattice parameter or slightly off position. This strongly suggests that gold substitutes zinc in both situations. This explains why no signal of crystalline gold is detected by XRD before the annealing step and why the crystallinity of $\mathrm{ZnO}$ is lowered upon gold incorporation.

As a summary of this section, it is observed that the incorporation of gold into $\mathrm{ZnO}$ and thermal annealing lead to strong changes in the microstructure of sputtered $\mathrm{ZnO}$ films. During deposition of $\mathrm{ZnO}-\mathrm{Au}$ films, gold is incorporated as individual or clustered atoms, most likely substituting zinc ones in the $\mathrm{ZnO}$ matrix. This decreases the crystallinity and introduces strong local strain in $\mathrm{ZnO}$. During annealing, gold atoms "trapped" in the $\mathrm{ZnO}$ matrix diffuse to form crystalline $\mathrm{Au}$ NPs with the fcc structure of gold dispersed in the nanocrystalline matrix and softening occurs. This is associated with a decrease in the overall in-plane compressive stress state and contraction of the $c$-axis parameter of $\mathrm{ZnO}$ to its bulk value.

3.2. Optical Properties. As shown previously, the incorporation of $\mathrm{Au}$ and the annealing treatment have a strong impact on the microstructure of the $\mathrm{ZnO}-\mathrm{Au}$ nanocomposite films. These factors are expected to affect the optical properties of the films. Localized surface plasmon resonance (LSPR) of isolated gold NPs can be detected in the visible range ${ }^{32}$ for different dielectric surrounding media. Its presence can be revealed by extracting the absorption coefficient from spectrophotometry measurements. The main purpose of this section is to study the influence of the microstructure of $\mathrm{ZnO}$ $\mathrm{Au}$ nanocomposite thin films on their LSPR behavior. It also provides information on the influence of the annealing step on the dielectric functions of components of the nanocomposite films, photoluminescence and bandgap behavior of the $\mathrm{ZnO}$ matrix.

Figure 5 plots the optical absorption for films with three different $\mathrm{Au}$ contents before and after annealing at 230 and 300

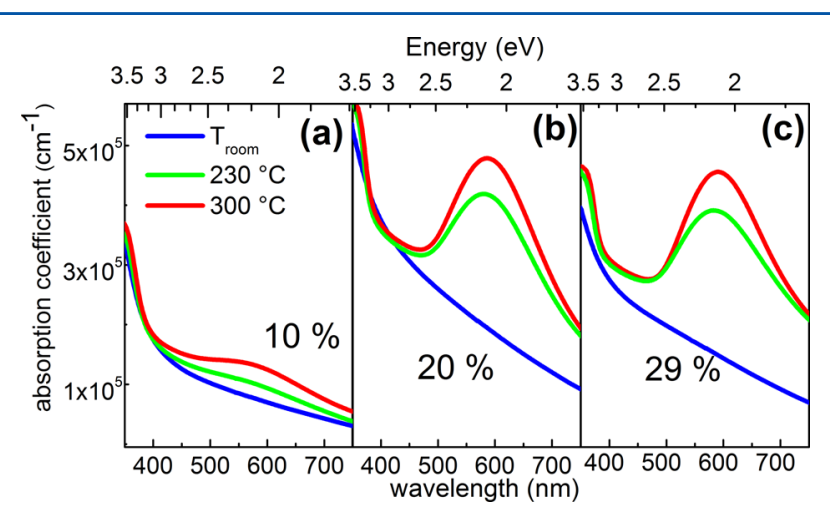

Figure 5. Absorption coefficient dispersion curves of $\mathrm{ZnO}-\mathrm{Au}$ nanocomposite thin films grown with different (a) $10 \%$, (b) $20 \%$, and (c) $29 \% \mathrm{Au}$ contents in the as-deposited state and after annealing at 230 and $300{ }^{\circ} \mathrm{C}$.

$\pm 5{ }^{\circ} \mathrm{C}$. For the as-deposited films (blue line), although some particles exhibit a mean diameter sufficient to stimulate observable LSPR absorption, there is no clear absorption band detected in the visible range, which is consistent with above conclusion that gold atoms substitute zinc as isolated atoms in the $\mathrm{ZnO}$ matrix or as precipitates in coherence with $\mathrm{ZnO}$ in as-deposited films. In such situation the electron density is probably too low to observe LSPR. The increase in absorption in the near UV corresponds to electronic transitions from the valence to conduction band of $\mathrm{ZnO}$. However, the characteristic near band edge absorption peak related to the generation of free excitons is not visible, likely due to a low crystallinity of the $\mathrm{ZnO}$ matrix. It is possible to observe a continuous absorption in the visible range referred in the following as background absorption. The absorption spectrum of pure gold films is dominated by valence electrons and the $5 \mathrm{~d}^{10} \rightarrow 6 \mathrm{sp}^{0}$ interband transition located around $\sim 1.8 \mathrm{eV}, 32,33$ but is not related to a sharp signal. It is typically associated with optical absorption below $600 \mathrm{~nm}$, contributing to the background absorption in the spectra of Figure 5. Other possible contributions correspond to absorption by defects in the matrix and a large plasmon band due to very small gold NPs.

Furthermore, Figure 5 informs us that the annealing process has two main consequences on the absorption properties of the coatings: (i) it promotes the development of the LSPR band, whose amplitude roughly depends on the gold content, and (ii) steepens the bandgap transition of the $\mathrm{ZnO}$ matrix. The development of LSPR is ascribed to the reorganization of gold atoms distributed in the $\mathrm{ZnO}$ matrix into $\mathrm{Au}$ NPs during annealing. Once developed, the LSPR band is centered near $590 \mathrm{~nm}$ and has a fwhm $\sim 170 \mathrm{~nm}$ for the highest gold loading. There is a difference with the LSPR band of isolated Au NP in air, centered around $539 \mathrm{~nm} .{ }^{32}$ The redshift observed with our films arises from a different dielectric function of the 
embedding medium compared to air, ${ }^{34,35}$ as clearly established by the Mie scattering theory.

While UV-vis spectrophotometry enables to measure the overall optical absorption of the nanocomposite films, the real and imaginary parts of the dielectric functions of the $\mathrm{ZnO}-\mathrm{Au}$ nanocomposite $\left(\varepsilon_{\mathrm{ZnO}-\mathrm{Au}-\mathrm{r}}\right.$ and $\left.\varepsilon_{\mathrm{ZnO}-\mathrm{Au}-\mathrm{i}}\right)$ and of the matrix ( $\varepsilon_{\text {matrix }(\mathrm{ZnO}) \text {-r }}$ and $\left.\varepsilon_{\text {matrix }(\mathrm{ZnO}) \text {-i }}\right)$ have been extracted from spectroscopic ellipsometry measurements of as-deposited and annealed films with a gold loading of $29 \%$ using a MaxwellGarnett effective medium. This is reported in Figure 6 together with the dielectric functions of pure $\mathrm{ZnO}$ films $\left(\varepsilon_{\mathrm{ZnO}-\mathrm{r}}\right.$ and $\varepsilon_{\mathrm{ZnO}-\mathrm{i}}$ grown and annealed under the same reactive conditions for comparison.
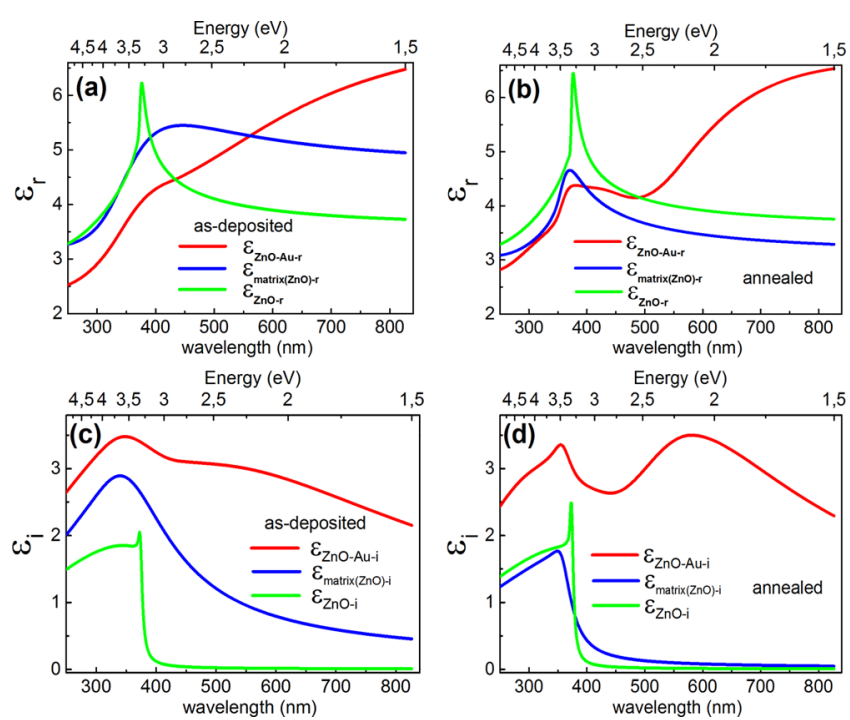

Figure 6. Real $(\mathrm{a}, \mathrm{b})$ and imaginary $(\mathrm{c}, \mathrm{d})$ dielectric functions of a $\mathrm{ZnO}$ $\mathrm{Au}$ nanocomposite film of $20 \% \mathrm{Au}$, its $\mathrm{ZnO}$ matrix, and pure $\mathrm{ZnO}$ films for the as-deposited state (a, c) and after annealing in air at 300 ${ }^{\circ} \mathrm{C}(\mathrm{b}, \mathrm{d})$.

The incorporation of gold modifies both real and imaginary dielectric functions of as-deposited coatings since higher absorption in the visible and less peaked excitonic transition are observed compared to pure $\mathrm{ZnO}$. This is also confirmed by looking at the dielectric functions of the zinc oxide matrix in the nanocomposite film. This can be explained by the poor crystallinity of the matrix and the presence of a large density of point defects in the as-deposited state. Without surprise, the near band edge region of $\varepsilon_{\mathrm{ZnO}-\mathrm{Au}-\mathrm{r}}$ and $\varepsilon_{\mathrm{ZnO} \text {-Au-i }}$ is dominated by the contribution of the zinc oxide matrix, while the visible range is dominated by the interband transitions of gold. Nevertheless, the visible range of $\varepsilon_{\mathrm{ZnO}-\mathrm{Au} \text {-i }}$ exhibits a bump we ascribe to a weak contribution of LSPR by gold NP. As seen in the previous section, all gold atoms are not in the form of metallic gold particles in the as-deposited state. Some metallic particles of very small size may be present though. In such case, intrinsic confinement effect induced by the scattering of free electrons on the NP surface has a strong impact on the LSPR band, explaining the large width and the small amplitude of the LSPR band. Annealing has a pronounced influence on the dielectric functions of pure $\mathrm{ZnO}$, the nanocomposite film and the zinc oxide matrix. Effectively, the matrix dielectric functions approach those of pure $\mathrm{ZnO}$ with a more peaked excitonic transition and steeper band edge. The optical quality of the matrix is unambiguously improved by annealing, which is consistent with its curing from gold atoms. The near band edge region of the effective dielectric functions also clearly manifests this improvement. Moreover, the visible region becomes dominated by LSPR. The volume fraction of gold inclusion in as-deposited and annealed films is estimated to $18.11 \pm$ $0.06 \%$ and $25.6 \pm 0.1 \%$. This is again consistent with the idea that a large number of gold atoms are not assembled into NPs in as-deposited films and diffuse toward pre-existing NPs upon thermal annealing. Moreover, NPs certainly change their structure and composition during annealing to reach those of pure gold as strongly supported by high resolution STEM analyzes. The bandgap was estimated from ellipsometry measurements. In the case of $\mathrm{ZnO}-\mathrm{Au}$ film, the band gap of the $\mathrm{ZnO}$ matrix was estimated from the Tauc plot. This method can be applied because, for $\mathrm{ZnO}-\mathrm{Au}$ film, the dielectric function of the matrix is not dominated by excitonic feature. The absorption coefficient of $\mathrm{ZnO}$ was calculated by using the dielectric function of the matrix determined by ellipsometry. The Tauc plot represented in Figure 7 was obtained by
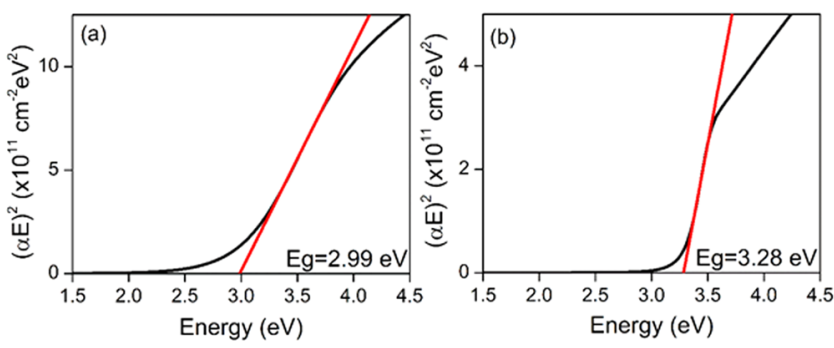

Figure 7. Tauc plot for extraction of the optical bandgap of asdeposited (a) and annealed at $300{ }^{\circ} \mathrm{C}$ (b) $\mathrm{ZnO}-\mathrm{Au}$ thin film containing $29 \% \mathrm{Au}$.

assuming the matrix as a direct semiconductor. The band gap energy of the $\mathrm{ZnO}$ matrix of as deposited and annealed $\mathrm{ZnO}$ $\mathrm{Au}$ films is estimated at 2.99 and $3.28 \mathrm{eV}$, respectively. The size of $\mathrm{ZnO}$ crystallites is little affected by annealing at such low temperature. Hence, exciton confinement can certainly not explain the observed difference in bandgap energies. In contrast, the presence of gold atoms in the nanocrystalline $\mathrm{ZnO}$ matrix could affect the measured bandgap energy through different effects. First, as suggested by Sahu et al., it could lead to broadening of the valence band resulting in bandgap narrowing. ${ }^{36}$ Second, it is evident from Figure 7 that strong band tailing occurs in the as-deposited state in line with the local distortion induced by the presence of isolated gold atoms in the $\mathrm{ZnO}$ matrix (see Figure 4). Positive hydrostatic strain is known to strongly reduce the bandgap $E_{\mathrm{g}}$ of $\mathrm{ZnO}$ by 11.75 $\varepsilon_{x x}{ }^{37}$ with $\varepsilon_{z z}=1.14 \varepsilon_{x x}, \varepsilon_{x x}$ and $\varepsilon_{z z}$ corresponding to strain along the $a$-axis and $c$-axis, respectively. Taking into account the stress state is nearly relaxed for $29 \% \mathrm{Au}$ (Figure $3 \mathrm{~b}$ ), we assume the zinc oxide lattice freely deforms upon incorporation of isolated $\mathrm{Au}$ atoms in as-deposited state. From data of Figure 3a, we calculate that $\varepsilon_{z z}$ and $\varepsilon_{x x}$ are close to $4.8 \times 10^{-3}$ and $4.2 \times$ $10^{-3} \% \mathrm{Au}^{-1}$, respectively. This corresponds to a theoretical reduction in bandgap energy of $27.5 \mathrm{meV}$. Since annealing was shown to cure the matrix from isolated $\mathrm{Au}$ atoms, the straininduced reduction in bandgap energy can be compared to the difference in bandgap energy of $28.1 \mathrm{meV}$ between annealed and as-deposited states. The values are very close, in favor to the interpretation that the change in bandgap energy during 
annealing results from relaxed distortions as the $\mathrm{ZnO}$ matrix is cured from gold atoms.

The Tauc plot method fails to determine the Band gap of pure $\mathrm{ZnO}$. Indeed, in this case strong excitonic effect is observed. The band gap energy and the exciton energy were directly estimated by using the Tanguy dispersion law. Both values are 3.37 and $0.05 \mathrm{eV}$, respectively. The blue-shift of the excitonic peak of the nanocomposite films compared to pure $\mathrm{ZnO}$ is explained by the confinement of excitons due to the nanocrystalline character of the $\mathrm{ZnO}$ matrix. Effectively, the confinement effect at the nanometer scale ${ }^{38,39}$ is known to increase the energy of the absorption edge.

PL measurements using $325 \mathrm{~nm}$ laser excitation have been performed on as-deposited films and the film containing $29 \%$ $\mathrm{Au}$ annealed at $300{ }^{\circ} \mathrm{C}$ in air, Figure 7 . Before annealing the near band edge (NBE) peak at the boundary between the UV and visible regions is extremely weak and the PL signal is dominated by a broad band in the visible range associated with electronic transitions involving point defect states. ${ }^{6}$ The signal increases upon gold incorporation, in line with recent reports on the optical properties of gold-doped $\mathrm{ZnO}$ nanorods. ${ }^{40}$ Annealing induces a strong rise of the overall PL signal. The NBE peak attributed to the recombination of free excitons develops which is in part explained by the curing of the matrix. Moreover, there is experimental evidence for the injection of "hot electrons" originated from plasmonic gold clusters in the conduction band of $\mathrm{ZnO}^{41}$ leading to an increase of the NBE emission. ${ }^{40}$ We also observe strong enhancement of the visible emission after annealing suggesting the possibility to excite the visible PL using LSPR as shown using plasmonic silver clusters on $\mathrm{ZnO}$ and silver or gold clusters separated from $\mathrm{ZnO}$ by a $\mathrm{MgO}$ buffer layer. ${ }^{42,43}$ The latter suggests that visible emission is stimulated by the local field associated with LSPR by gold NPs. Careful observation of the shape of absorption and emission curves of Figure 8 strongly supports this hypothesis.

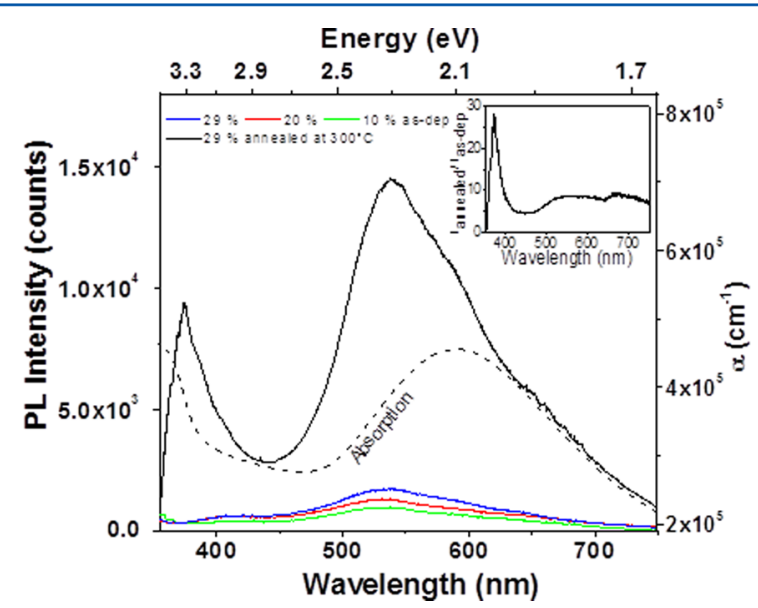

Figure 8. Photoluminescence of as-deposited films and film containing $29 \% \mathrm{Au}$ annealed at $300{ }^{\circ} \mathrm{C}$. The inset shows the ratio of PL intensity of the film containing $29 \% \mathrm{Au}$ after annealing to the PL intensity before annealing for the same sample.

Effectively, emission and absorption curve shapes follow the very same evolution above $640 \mathrm{~nm}$ for the sample containing $29 \% \mathrm{Au}$ after annealing. Moreover, the ratio of PL intensity after annealing to the intensity before annealing for the same sample (inset of Figure 8) exhibits a step at $640 \mathrm{~nm}$. The PL signal in this wavelength region is usually referred as orange-red contribution. It has not been clearly ascribed so far but could correspond to electronic transition to an oxygen interstitial acceptor state in $\mathrm{ZnO}^{6}$

\section{CONCLUSIONS}

We have shown using X-ray diffraction and high resolution scanning transmission electron microscopy that $\mathrm{ZnO}-\mathrm{Au}$ nanocomposite films consisting in gold nanoparticles embedded by a zinc oxide matrix can be easily synthesized using reactive magnetron sputtering of gold and zinc sources followed by annealing in air. In the as-deposited state gold substitutes zinc in $\mathrm{ZnO}$ as isolated or grouped atoms forming precipates in coherence with the crystal lattice of $\mathrm{ZnO}$. Moreover, strong localized surface plasmon resonance absorption triggered by the formation of gold nanoparticles and curing of the matrix can be achieved by a thermal annealing step at moderate temperature in air. In parallel, photoluminescence is strongly enhanced both at the near band edge and in the visible regions we interpreted by the emission of "hot electrons" and coupling of emission by point defects to the local field associated with LSPR. This simple synthesis route could lead to the application of $\mathrm{ZnO}-\mathrm{Au}$ nanocomposite in new generations of photodiodes, solar cells, and metamaterials.

\section{ASSOCIATED CONTENT}

\section{Supporting Information}

The Supporting Information is available free of charge on the ACS Publications website at DOI: 10.1021/acs.jpcc.6b09974.

Effective medium model applied to spectroscopic ellipsometry measurements; X-ray diffraction; Transmission electron micrographs; and Filtered Fourier transform (PDF).

\section{AUTHOR INFORMATION}

\section{Corresponding Author}

*E-mail: david.horwat@univ-lorraine.fr. Phone: +33 (0)3 8358 4252.

ORCID

David Horwat: 0000-0001-7938-7647

\section{Author Contributions}

The manuscript was written through contributions of all authors. All authors have given approval to the final version of the manuscript.

\section{Funding}

Erasmus Mundus scholarship was awarded to W.C. to conduct his Ph.D. thesis within the DocMASE program.

Notes

The authors declare no competing financial interest.

\section{ACKNOWLEDGMENTS}

Authors acknowledge Patrice Miska (IJL) for technical help with setting up PL measurements.

\section{REFERENCES}

(1) Editorial. Surface plasmon resurrection. Nat. Photonics 2012, 6, 707.

(2) Nan, F.; Liang, S.; Wang, J.-H.; Liu, X.-L.; Yang, D.-J.; Yu, X.-F.; Zhou, L.; Hao, Z.-H.; Wang, Q.-Q. Semiconductor-metal nanocomposites. photoinduced fusion and photocatalysis of gold-capped $\mathrm{TiO}_{2}\left(\mathrm{TiO}_{2} /\right.$ Gold $)$ nanoparticles. J. Phys. Chem. B 2001, 105, 960966. 
(3) Freund, H.-J.; Nilius, N.; Risse, T.; Schauermann, S. A fresh look at an old nano-technology: catalysis. Phys. Chem. Chem. Phys. 2014, 16, $8148-8167$.

(4) Ohodnicki, P. R., Jr.; Buric, M. P.; Brown, T. D.; Matranga, C.; Wang, C.; Baltrus, J.; Andio, M. Plasmonic nanocomposite thin film enabled fiber optic sensors for simultaneous gas and temperature sensing at extreme temperatures. Nanoscale 2013, 5, 9030-9039.

(5) Mickan, M.; Helmersson, U.; Rinnert, H.; Ghanbaja, J.; Muller, D.; Horwat, D. Room temperature deposition of homogeneous, highly transparent and conductive $\mathrm{Al}$-doped $\mathrm{ZnO}$ films by reactive high power impulse magnetron sputtering. Sol. Energy Mater. Sol. Cells 2016, 157, 742-749.

(6) Chamorro, W.; Horwat, D.; Pigeat, P.; Miska, P.; Migot, S.; Soldera, F.; Boulet, P.; Mücklich, F. Near-room temperature singledomain epitaxy of reactively sputtered $\mathrm{ZnO}$ films. J. Phys. D: Appl. Phys. 2013, 46, 235107.

(7) Jullien, M.; Horwat, D.; Manzeh, F.; Escobar Galindo, R.; Bauer, Ph.; Pierson, J.-F.; Endrino, J.-L. Influence of the nanoscale structural features on the properties and electronic structure of Al-doped $\mathrm{ZnO}$ thin films: an X-ray absorption study. Sol. Energy Mater. Sol. Cells 2011, 95, 2341-2346.

(8) Gogurla, N.; Sinha, A. K.; Santra, S.; Manna, S.; Ray, S. K. Multifunctional $\mathrm{Au}-\mathrm{ZnO}$ plasmonic nanostructures for enhanced UV photodetector and room temperature NO sensing devices. Sci. Rep. 2014, 4, 6483.

(9) Della Gaspera, E.; Guglielmi, M.; Martucci, A.; Giancaterini, L.; Cantalini, C. Enhanced optical and electrical gas sensing response of sol-gel based $\mathrm{NiO}-\mathrm{Au}$ and $\mathrm{ZnO}-\mathrm{Au}$ nanostructured thin films. Sens. Actuators, B 2012, 164, 54-63.

(10) Della Gaspera, E.; Guglielmi, M.; Perotto, G.; Agnoli, S.; Granozzi, G.; Post, M. L.; Martucci, A. CO optical sensing properties of nanocrystalline $\mathrm{ZnO}-\mathrm{Au}$ films: Effect of doping with transition metal ions. Sens. Actuators, B 2012, 161 (1), 675-683.

(11) Wang, C.; Ranasingha, O.; Natesakhawat, S.; Ohodnicki, P. R., Jr.; Andio, M.; Lewis, J. P.; Matrang, C. Visible light plasmonic heating of $\mathrm{Au}-\mathrm{ZnO}$ for the catalytic reduction of $\mathrm{CO}_{2}$. Nanoscale 2013, 5, 6968-6974.

(12) Li, Y.; Zhang, B.-P.; Zhao, J.-X. Enhanced photocatalytic performance of $\mathrm{Au}-\mathrm{Ag}$ alloy modified $\mathrm{ZnO}$ nanocomposite films. $J$. Alloys Compd. 2014, 586, 663-668.

(13) Clavero, C. Plasmon-induced hot-electron generation at nanoparticle/metal-oxide interfaces for photovoltaic and photocatalytic devices. Nat. Photonics 2014, 8, 95-103.

(14) Knight, M. W.; Sobhani, H.; Nordlander, P.; Halas, N. J. Photodetection with active optical antennas. Science 2011, 332, 702704.

(15) Linic, L. S.; Christopher, P.; Ingram, D. B. Plasmonic-metal nanostructures for efficient conversion of solar to chemical energy. Nat. Mater. 2011, 10, 911-921.

(16) Knight, M. W.; Wang, Y.; Urban, A. S.; Sobhani, A.; Zheng, B. Y.; Nordlander, P.; Halas, N. J. Embedding plasmonic nanostructure diodes enhances hot electron emission. Nano Lett. 2013, 13, 1687.

(17) White, T. P.; Catchpole, K. R. Plasmon-enhanced internal photoemission for photovoltaics: theoretical efficiency limits. Appl. Phys. Lett. 2012, 101, 13707-137015.

(18) Yoshino, T.; Takanezawa, S.; Ohmori, T.; Masuda, H. Preparation of $\mathrm{ZnO} / \mathrm{Au}$ nanocomposite thin films by electrodeposition. Jpn. J. Appl. Phys. Part 2-Lett. 1996, 35 (11B), L1512L1514.

(19) Pal, U.; García-Serrano, J.; Casarrubias-Segura, G.; Koshizaki, N.; Sasaki, T.; Terahuchi, S. Structure and optical properties of M/ $\mathrm{ZnO}(\mathrm{M}=\mathrm{Au}, \mathrm{Cu}, \mathrm{Pt})$ nanocomposites. Sol. Energy Mater. Sol. Cells 2004, 81, 339-348.

(20) Bajaj, G.; Soni, R. K. Nanocomposite $\mathrm{ZnO} / \mathrm{Au}$ formation by pulsed laser irradiation. Appl. Surf. Sci. 2010, 256, 6399-6402.

(21) Horwat, D.; Pierson, J.-F.; Billard, A. Magnetron sputtering of NASICON $\left(\mathrm{Na}_{3} \mathrm{Zr}_{2} \mathrm{Si}_{2} \mathrm{PO}_{12}\right)$ thin films PART II: A novel approach. Surf. Coat. Technol. 2007, 201, 7060-7065.
(22) Mezin, A. Coating internal stress measurement through the curvature method: A geometry-based criterion delimiting the relevance of Stoney's formula. Surf. Coat. Technol. 2006, 200, 5259-5267.

(23) Tangui, C. Optical dispersion by Wannier excitons. Phys. Rev. Lett. 1995, 75, 4090.

(24) Palik, E. D. Handbook of Optical Constants of Solids; Academic Press: Orlando, 1985.

(25) Battie, Y.; Resano-Garcia, A.; Chaoui, N.; Zhang, Y.; En Naciri, A. Extended Maxwell-Garnett-Mie formulation applied to size dispersion of metallic nanoparticles embedded in host liquid matrix. J. Chem. Phys. 2014, 140, 044705.

(26) Xu, Y.; Yao, B.; Li, Y. F.; Ding, Z. H.; Li, J. C.; Wang, H. Z.; Zhang, Z. Z.; Zhang, L. G.; Zhao, H. F.; Shen, D. Z. Chemical states of gold doped in $\mathrm{ZnO}$ films and its effect on electrical and optical properties. J. Alloys Compd. 2014, 585, 479-484.

(27) Barna, P. B.; Adamik, M. Fundamental structure forming phenomena of polycrystalline films and the structure zone models. Thin Solid Films 1998, 317, 27-33.

(28) Gries, T.; Catrin, R.; Migot, S.; Soldera, F.; Endrino, J.-L.; Landa-Canovas, A. R.; Cleymand, F.; Mangin, D.; Mücklich, F.; Horwat, D. Local Modification of the microstructure and electrical properties of multifunctional $\mathrm{Au}-\mathrm{YSZ}$ nanocomposite thin films by laser interference patterning. ACS Appl. Mater. Interfaces 2014, 6, 13707-13715.

(29) Jogai, B. Effect of in-plane biaxial strains on the band structure of wurtzite GaN. Phys. Rev. B: Condens. Matter Mater. Phys. 1998, 57, 2382.

(30) Morkoç, H.; Özgür, U. Zinc Oxide: Fundamentals, Materials and Device Technology; Wiley-VCH: Weinheim, 2009.

(31) Voevodin, A. A.; Hu, J. J.; Fitz, T. A.; Zabinski, J. S. Tribological properties of adaptive nanocomposite coatings made of yttria stabilized zirconia and gold. Surf. Coat. Technol. 2001, 146-147, 351-356.

(32) Alvarez, M. M.; Khoury, J. T.; Schaaff, T. G.; Shafigullin, M. N.; Vezmar, I.; Whetten, R. L. Optical absorption spectra of nanocrystal gold molecules. J. Phys. Chem. B 1997, 101, 3706-3712.

(33) Kreibig, P. D. U.; Vollmer, P. D. M. Optical Properties of Metal Clusters, Springer Series in Materials Science; Springer: Berlin, Heidelberg, 1995; pp 13-201.

(34) Cottancin, E.; Celep, G.; Lermé, J.; Pellarin, M.; Huntzinger, J. R; Vialle, J. L.; Broyer, M. Theor. Chem. Acc. 2006, 116 (4-5), 514523.

(35) Carmina Monreal, R.; Antosiewicz, T. J.; Apell, S. P. Competition between surface screening and size quantization for surface plasmons in nanoparticles. New J. Phys. 2013, 15, 083044.

(36) Sahu, D.; Panda, N. R.; Acharya, B. S.; Panda, A. K. Enhanced UV absorbance and photoluminescence properties of ultrasound assisted synthesized gold doped $\mathrm{ZnO}$ nanorods. Opt. Mater. 2014, 36, $1402-1407$.

(37) Hanada, T. Basic Properties of $\mathrm{ZnO}, \mathrm{GaN}$, and Related Materials; Oxide and Nitride Semiconductors. Advances in Materials Research; Springer: Berlin, Heidelberg, 2009; Vol. 12, pp 1-18.

(38) Kosacki, I.; Anderson, H. U. Microstructure - Property relationships in nanocrystalline oxide thin films. Ionics 2000, 6, 294311.

(39) Mosquera, A. A.; Horwat, D.; Rashkovskiy, A.; Kovalev, A.; Miska, P.; Wainstein, D.; Albella, J. M.; Endrino, J. L. Exciton and core-level electron confinement effects in transparent $\mathrm{ZnO}$ thin films. Sci. Rep. 2013, 3, 1714.

(40) Viter, R.; et al. The influence of localized plasmons on the optical properties of $\mathrm{Au} / \mathrm{ZnO}$ nanostructures. J. Mater. Chem. C 2015, 3, 6815-6821.

(41) Chen, Z. H.; et al. Vertically aligned $\mathrm{ZnO}$ nanorod arrays sentisized with gold nanoparticles for schottky barrier photovoltaic cells. J. Phys. Chem. C 2009, 113, 13433-13437.

(42) Cheng, P.; Li, D.; Yuan, Z.; Chen, P.; Yang, D. Enhancement of $\mathrm{ZnO}$ light emission via coupling with localized surface plasmon of $\mathrm{Ag}$ island film. Appl. Phys. Lett. 2008, 92, 041119. 
(43) Lawrie, B. J.; Haglund, R. F., Jr; Mu, R. Enhancement of $\mathrm{ZnO}$ photoluminescence by localized and propagating surface plasmons. Opt. Express 2009, 17, 2565-2572. 